UDC 517.9

\title{
NECESSARY CONDITIONS FOR EXISTENCE OF POSITIVE SOLUTIONS OF SECOND ORDER LINEAR DIFFERENCE EQUATIONS AND SUFFICIENT CONDITIONS FOR OSCILLATION OF SOLUTIONS
}

\section{НЕОБХІДНІ УМОВИ ІСНУВАННЯ ДОДАТНИХ РОЗВ'ЯЗКІВ ЛІНІЙНИХ РІЗНИЦЕВИХ РІВНЯНЬ ДРУГОГО ПОРЯДКУ \\ ТА ДОСТАТНІ УМОВИ ДЛЯ КОЛИВАННЯ РОЗВ'ЯЗКІВ}

\section{R. Koplatadze}

I. Javakhishvili Tbilisi State Univ.

2, University St., Tbilisi 0143, Georgia

e-mail: r_koplatadze@yahoo.com

\section{G. Kvinikadze}

A. Razmadze Math. Inst.

1, M. Aleksidze St., Tbilisi 0193, Georgia

e-mail: giokvi@rmi.acnet.ge

The difference equation

$$
\Delta^{2} u(k)+\sum_{l=1}^{m} p_{l}(k) u\left(\tau_{l}(k)\right)=0,
$$

is considered, where $m \in N$, the functions $p_{l}: N \rightarrow R_{+}, \tau_{l}: N \rightarrow N, \lim _{k \rightarrow+\infty} \tau_{l}(k)=+\infty, l=1, \ldots, m$, are defined on the set of natural numbers and the difference operator is defined by $\Delta u(k)=u(k+1)-u(k)$, $\Delta^{2}=\Delta \circ \Delta$.

Necessary conditions are obtained for the above equation to have a positive solution. Besides, oscillation criteria of a new type are obtained generalizing some earlier known results.

Розглянуто різницеве рівняння

$$
\Delta^{2} u(k)+\sum_{l=1}^{m} p_{l}(k) u\left(\tau_{l}(k)\right)=0,
$$

де $m \in N$, функциї $p_{l}: N \rightarrow R_{+}, \tau_{l}: N \rightarrow N, \lim _{k \rightarrow+\infty} \tau_{l}(k)=+\infty, l=1, \ldots, m$, визначено на множині натуральних чисел, а різницевий оператор $\Delta u(k)=u(k+1)-u(k), \Delta^{2}=\Delta \circ \Delta$.

Встановлено необхідні умови для того, щеоб це рівняння мало додатний розв'язок. Також отримано критерії нового типу, які узагальнюють попередні результати, для існування коливання.

1. Introduction. Consider the difference equation

$$
\Delta^{2} u(k)+\sum_{l=1}^{m} p_{l}(k) u\left(\tau_{l}(k)\right)=0,
$$

(C) R. Koplatadze, G. Kvinikadze, 2009 
where $m \geq 1$ is a natural number, $p_{j}: N \rightarrow R_{+}, \tau_{j}: N \rightarrow N, j=1, \ldots, m$, are functions defined on the set of natural numbers $N=\{1,2, \ldots\}, \Delta u(k)=u(k+1)-u(k)$ and $\Delta^{2}=\Delta \circ \Delta$. Everywhere below it is assumed that

$$
\begin{aligned}
& \lim _{k \rightarrow+\infty} \tau_{l}(k)=+\infty, \quad l=1, \ldots, m \\
& \sup \left\{p_{l}(i): i \geq k\right\}>0 \text { for } \quad k \in N, \quad l=1, \ldots, m .
\end{aligned}
$$

For each $n \in N$ denote $N_{n}=\{n, n+1, \ldots\}$.

Definition 1.1. Let $n \in N$. We will call a function $u: N \rightarrow R$ a proper solution of the equation (1.1) on the set $N_{n}$, if it satisfies (1.1) on $N_{n}$ and $\sup \{|u(i)|: i \geq k\}>0$ for any $k \in N_{n}$.

Definition 1.2. We say that a proper solution $u: N_{n} \rightarrow R$ of the equation (1.1) is oscillatory if for any $k \in N_{n}$ there are $n_{1}, n_{2} \in N_{k}$ such that $u\left(n_{1}\right) u\left(n_{2}\right) \leq 0$. Otherwise the solution is called nonoscillatory.

In Section 3 of the presented paper a necessary condition for existence of a positive solution of the equation (1.1) is obtained. Using that result, in Section 4 sufficient conditions for oscillation of all solutions of (1.1) are given which generalizes the results presented in [1], where $\liminf _{k \rightarrow+\infty} \frac{\tau_{l}(k)}{k}>0, l=1, \ldots, m$, was an essential assumption. In the given paper this restriction, in general, is removed.

The problem of oscillation of solutions of the equation of the type (1.1) has been studied by several authors, see e.g. [2-10] and the references therein. Everywhere below it is assumed that the conditions

$$
\sum_{k=1}^{+\infty} k\left(\sum_{l=1}^{m} p_{l}(k)\right)=+\infty
$$

and

$$
\sum_{k=1}^{+\infty}\left(\sum_{l=1}^{m} \tau_{l}(k) p_{l}(k)\right)=+\infty
$$

are fulfilled.

Using the fixed point principle, one can easily show that the conditions (1.4) and (1.5) are necessary for oscillation of all solutions of the equation (1.1) [1].

\section{Some auxiliary statements.}

Lemma 2.1. Let $\left\{a_{i}\right\}_{i=1}^{+\infty},\left\{b_{i}\right\}_{i=1}^{+\infty}$ be two infinite sequences of real numbers, the series $\sum_{i=1}^{+\infty} b_{i}$ be convergent and $a_{i} B_{i+1} \rightarrow 0$ as $i \rightarrow+\infty$, where $B_{i}=\sum_{j=i}^{+\infty} b_{j}$. Then the convergence of either of the series $\sum_{i=1}^{+\infty} a_{i} b_{i}$ and $\sum_{i=2}^{+\infty}\left(a_{i}-a_{i-1}\right) B_{i}$ implies the convergence of the other and

$$
\sum_{i=1}^{+\infty} a_{i} b_{i}=a_{1} B_{1}+\sum_{i=2}^{+\infty}\left(a_{i}-a_{i-1}\right) B_{i} .
$$


Lemma 2.2. Let $u: N_{n} \rightarrow R$ be a nonoscillatory proper solution of (1.1). Then there exists $k_{0} \in N_{n}$ such that

$$
u(k) \Delta u(k)>0 \quad \text { for } \quad k \in N_{k_{0}} .
$$

Lemma 2.3. Suppose that (1.4) and (1.5) hold and $u: N_{n} \rightarrow R$ is a nonoscillatory solution of (1.1). Then

$$
\lim _{k \rightarrow+\infty} u(k)=+\infty, \quad \limsup _{k \rightarrow+\infty} \frac{|u(k)|}{k}<+\infty
$$

Lemma 2.4. Let $\varphi, \psi: N \rightarrow(0,+\infty), \psi$ be nonincreasing and

$$
\begin{aligned}
& \lim _{k \rightarrow+\infty} \varphi(k)=+\infty, \\
& \liminf _{k \rightarrow+\infty} \psi(k) \widetilde{\varphi}(k)=0,
\end{aligned}
$$

where $\widetilde{\varphi}(k)=\inf \{\varphi(s): s \geq k, s \in N\}$. Then there exists an increasing sequence of natural numbers $\left\{k_{i}\right\}_{i=1}^{+\infty}$ such that

$$
\widetilde{\varphi}\left(k_{i}\right)=\varphi\left(k_{i}\right), \quad \psi(k) \widetilde{\varphi}(k) \geq \psi\left(k_{i}\right) \widetilde{\varphi}\left(k_{i}\right), \quad k=1,2, \ldots, k_{i}, \quad i=1,2, \ldots
$$

We refer the reader to [1] for the proofs of Lemmas 2.1-2.4.

Lemma 2.5. Let $\tau_{l}: N \rightarrow N, l=1, \ldots, m$, and (1.2) be fulfilled. Then there exists a nondecreasing function $\sigma: N \rightarrow N$ such that

$$
\begin{aligned}
& \text { 1) } \lim _{k \rightarrow+\infty} \sigma(k)=+\infty, \\
& \text { 2) } \sigma(k) \leq \min \left\{k, \tau_{l}(k): l=1, \ldots, m\right\}, \\
& \text { 3) } \sigma\left(N_{k}\right) \supset U_{l=1}^{m} \tau_{l}\left(N_{k}\right) \quad \text { for any } k \in N .
\end{aligned}
$$

Proof. Consider the sequence

$$
\begin{aligned}
A= & \left\{a_{1}, a_{2}, \ldots, a_{2 m+2}, \ldots\right\}= \\
& =\left\{1, \tau_{1}(1), \ldots, \tau_{m}(1), 2, \tau_{1}(2), \ldots, \tau_{m}(2), \ldots\right\}
\end{aligned}
$$

and denote by $\tau$ the function $\tau: N \rightarrow A$ thus defined. By (1.2) it is obvious that

$$
\begin{gathered}
\lim _{k \rightarrow+\infty} \tau(k)=+\infty \quad \text { and } \tau\left(N_{k}\right) \supset \tau_{l}\left(N_{k}\right), \quad l=1, \ldots, m, \\
\text { for any } k \in N .
\end{gathered}
$$

Introduce the following sets

$$
\begin{aligned}
& s \in A_{1} \Leftrightarrow s \in N, \quad \tau(s)=\inf \{\tau(k) ; k \in N\}, \\
& s \in A_{j} \Leftrightarrow s \in N, \quad \tau(s)=\inf \left\{\tau(k) ; k \in N \backslash U_{i=1}^{j-1} A_{i}\right\}, \quad j=2,3, \ldots,
\end{aligned}
$$


and denote $\xi_{j}=\max A_{j}, j=1,2, \ldots, \xi_{1}^{0}=\xi_{1}, \xi_{j}^{0}=\max \left\{\xi_{j}, \xi_{j-1}^{0}+1\right\}, j=2,3, \ldots \mathrm{We}$ will construct the function $\sigma$ as follows: $\sigma(k)=\tau\left(\xi_{1}\right)$ for $1 \leq k \leq \xi_{1}^{0}, \sigma(k)=\tau\left(\xi_{j}\right)$ for $\xi_{j-1}^{0}<k \leq \xi_{j}^{0}, j=2,3, \ldots$. The function $\sigma$ is obviously nondecreasing and satisfies the conditions 1 and 2. We also have $\sigma\left(N_{k}\right) \supset \tau\left(N_{k}\right)$ for any $k \in N$. Therefore in view of (2.6) it is obvious that the condition 3 is also satisfied.

The lemma is proved.

3. A necessary condition for the existence of a positive solution. The result obtained in this section is very important for establishing sufficient conditions of oscillation of all proper solutions of the equation (1.1). Below the following notation will be used.

Let $k_{0} \in N$. Denote by $U_{k_{0}}$ the set of all proper solutions of (1.1) satisfying $u(k)>0$ for $k \in N_{k_{0}}$.

Theorem 3.1. Let $k_{0} \in N, U_{k_{0}} \neq \varnothing$. Then there exists $\lambda \in[0,1]$ such that

$$
\limsup _{\varepsilon \rightarrow 0+}\left(\liminf _{k \rightarrow+\infty} \rho(k, \varepsilon, \lambda)\right) \leq 1
$$

where

$$
\begin{aligned}
& \rho(k, \varepsilon, \lambda)=k^{-\lambda-h_{2 \varepsilon}(\lambda)} \sum_{i=1}^{k-1}(\sigma(i))^{h_{1 \varepsilon}(\lambda)+h_{2 \varepsilon}(\lambda)} \sum_{j=i}^{+\infty}\left(\sum_{l=1}^{m} p_{l}(j)\left(\tau_{l}(j)\right)^{\lambda-h_{1 \varepsilon}(\lambda)}\right) \\
& h_{1 \varepsilon}(\lambda)=\left\{\begin{array}{ll}
0, & \text { if } \lambda=0, \\
\varepsilon, & \text { if } \lambda \in(0,1],
\end{array} \quad h_{2 \varepsilon}(\lambda)= \begin{cases}0, & \text { if } \lambda=1, \\
\varepsilon, & \text { if } \lambda \in[0,1),\end{cases} \right.
\end{aligned}
$$

and $\sigma$ is any function satisfying (2.5) (such a function exists due to Lemma 2.5).

Proof. First of all note that according to Lemma 2.5 there exists a function $\sigma$ satisfying the conditions (2.5). Let $k_{0} \in N$ and $U_{k_{0}} \neq \varnothing$. Show that there is a $\lambda_{0} \in[0,1]$ such that for $\lambda=\lambda_{0}$ the inequality (3.1) is fulfilled, where the function $\rho$ is given by (3.2) and (3.3). By definition of the set $U_{k_{0}}$, the equation (1.1) has a solution $u$ satisfying the condition $u: N_{k_{0}} \rightarrow(0,+\infty)$. Lemmas 2.2 and 2.3 obviously imply that

$$
u(k) \uparrow+\infty \text { and } \frac{u(k)}{k} \text { for } k \uparrow+\infty,
$$

and

$$
u(k) \geq \sum_{i=k_{1}}^{k-1} \sum_{j=i}^{+\infty}\left(\sum_{l=1}^{m} p_{l}(j) u\left(\tau_{l}(j)\right)\right), \quad k=k_{1}+1, \quad k_{1}+2, \ldots,
$$

where $k_{1} \in N_{k_{0}}$ is sufficiently large.

Denote

$$
\Lambda_{u}=\left\{\lambda \in[0,1]: \lim _{k \rightarrow+\infty} \frac{u(k)}{k^{\lambda}}=+\infty\right\}, \quad u \in U_{k_{0}}
$$

(if there is no $\lambda \in[0,1]$ such that $\lim _{k \rightarrow+\infty} \frac{u(k)}{k^{\lambda}}=+\infty$, then we assume that $\Lambda_{u}=\varnothing$ ). 
By (3.4) we obviously have $0 \in \Lambda_{u}$ and $1 \notin \Lambda_{u}$. Therefore

$$
\Lambda_{u} \subset[0,1) \quad \text { and } \quad \lambda_{0}=\sup \Lambda_{u} \in[0,1]
$$

Show that the $\lambda_{0}$ selected in this way satisfies (3.1) $\left(\lambda=\lambda_{0}\right)$. First show that

$$
\frac{u(k)}{k} \downarrow 0 \quad \text { for } \quad k \uparrow+\infty .
$$

Indeed, if this is not the case, then by Lemma 2.2, $c>0$ may be found such that $u\left(\tau_{l}(i)\right) \geq$ $\geq c \tau_{l}(i), l=1, \ldots, m$, for $i \in N_{k_{0}}$, where $k_{0}$ is sufficiently large. Therefore in view of (1.5) from (1.1) we will have

$$
\Delta(u(k)) \geq \sum_{j=k_{0}}^{+\infty}\left(\sum_{l=1}^{m} p_{l}(j) u_{l}\left(\tau_{l}(j)\right)\right) \geq c \sum_{j=k_{0}}^{+\infty}\left(\sum_{l=1}^{m} p_{l}(j) \tau_{l}(j)\right)=+\infty
$$

The obtained contradiction shows that (3.6) is true. In view of (3.3), (3.6) and the choice of $\lambda_{0}$, for all sufficiently small $\varepsilon$ we have

$$
\begin{gathered}
\lim _{k \rightarrow+\infty} \frac{u(k)}{k^{\lambda_{0}-h_{1 \varepsilon}\left(\lambda_{0}\right)}}=+\infty, \quad \liminf _{k \rightarrow+\infty} \frac{u(k)}{k^{\lambda_{0}+h_{2 \varepsilon}\left(\lambda_{0}\right)}}=0 \\
0 \leq \lambda_{0}-h_{1 \varepsilon}\left(\lambda_{0}\right)<\lambda_{0}+h_{2 \varepsilon}\left(\lambda_{0}\right) \leq 1
\end{gathered}
$$

Denote

$$
\widetilde{\varphi}(k)=\inf \left\{\frac{u(\sigma(s))}{(\sigma(s))^{\lambda_{0}-h_{1 \varepsilon}\left(\lambda_{0}\right)}}: s \geq k \geq k_{0}, s \in N\right\} .
$$

For all sufficiently small positive $\varepsilon$, due to (3.8) and (3.9), the condition

$$
\liminf _{k \rightarrow+\infty} \frac{\widetilde{\varphi}(k)}{(\sigma(k))^{h_{1 \varepsilon}\left(\lambda_{0}\right)+h_{2 \varepsilon}\left(\lambda_{0}\right)}}=0
$$

is fulfilled. Indeed, for all sufficiently small $\varepsilon$ in view of (3.8) and (3.9) we have

$$
\frac{\widetilde{\varphi}(k)}{(\sigma(k))^{h_{1 \varepsilon}\left(\lambda_{0}\right)+h_{2 \varepsilon}\left(\lambda_{0}\right)}} \leq \frac{u(\sigma(k))}{(\sigma(k))^{\lambda_{0}+h_{2 \varepsilon}\left(\lambda_{0}\right)}}
$$

Hence the second condition of (3.7) implies (3.10). Since $\lim _{k \rightarrow+\infty} \sigma(k)=+\infty$, due to (3.5) there exists $k_{2} \in N_{k}$ such that

$$
u(\sigma(k)) \geq \sum_{i=k_{1}}^{\sigma(k)-1} \sum_{j=i}^{+\infty}\left(\sum_{l=1}^{m} p_{l}(j) u\left(\tau_{l}(j)\right)\right), \quad k \in N_{k_{2}}
$$


We see that (3.7), (3.8) and (3.10) obviously imply that the functions

$$
\varphi(k)=\frac{u(\sigma(k))}{(\sigma(k))^{\lambda_{0}-h_{1 \varepsilon}\left(\lambda_{0}\right)}}, \quad \psi(k)=(\sigma(k))^{-\left(h_{1 \varepsilon}\left(\lambda_{0}\right)+h_{2 \varepsilon}\left(\lambda_{0}\right)\right)}
$$

satisfy the conditions of Lemma 2.4. Therefore there exists a sequence $\left\{k_{i}\right\}_{i=3}^{+\infty}$ such that $k_{i} \in$ $\in N_{k_{2}}, i=3,4, \ldots, k_{i} \uparrow+\infty$ as $i \uparrow+\infty$ and

$$
\begin{aligned}
& \widetilde{\varphi}\left(k_{i}\right)=\varphi\left(k_{i}\right), \quad i=3,4, \ldots, \\
& \psi\left(k_{i}\right) \widetilde{\varphi}\left(k_{i}\right) \leq \psi(s) \widetilde{\varphi}(s), \quad k_{2} \leq s \leq k_{i}, \quad s \in N, \quad i=3,4, \ldots
\end{aligned}
$$

Since the function $\sigma$ satisfies (2.5), it is obvious that for any sufficiently large $k \in N$ and sufficiently small $\varepsilon>0$

$$
\begin{gathered}
\inf \left\{\frac{u\left(\tau_{l}(s)\right)}{\left(\tau_{l}(s)\right)^{\lambda_{0}-h_{1 \varepsilon}\left(\lambda_{0}\right)}}: s \geq k\right\} \geq \inf \left\{\frac{u(\sigma(s))}{(\sigma(s))^{\lambda_{0}-h_{1 \varepsilon}\left(\lambda_{0}\right)}}: s \geq k\right\}=\widetilde{\varphi}(k), \\
l=1, \ldots, m .
\end{gathered}
$$

Therefore, taking into account the nondecreasing character of $\widetilde{\varphi}(k)$, from (3.11) we obtain

$$
u(\sigma(k)) \geq \sum_{i=k_{2}}^{\sigma(k)-1} \widetilde{\varphi}(i) \sum_{j=i}^{+\infty}\left(\sum_{l=1}^{m} p_{l}(j)\left(\tau_{l}(j)\right)^{\lambda_{0}-h_{1 \varepsilon}\left(\lambda_{0}\right)}\right)
$$

Hence, with regard to (3.14) we get

$$
u\left(\sigma\left(k_{i}\right)\right) \geq \widetilde{\varphi}(k) \psi\left(k_{i}\right) \sum_{j=k_{2}}^{\sigma\left(k_{i}\right)-1}(\sigma(j))^{h_{1 \varepsilon}\left(\lambda_{0}\right)+h_{2 \varepsilon}\left(\lambda_{0}\right)} \sum_{s=j}^{+\infty}\left(\sum_{l=1}^{m} p_{l}(s)\left(\tau_{l}(s)\right)^{\lambda_{0}-h_{1 \varepsilon}\left(\lambda_{0}\right)}\right), i=3,4, \ldots
$$

By (3.12) and (3.13) we have

$$
\limsup _{i \rightarrow+\infty}\left(\sigma\left(k_{i}\right)\right)^{-\lambda_{0}-h_{2 \varepsilon}\left(\lambda_{0}\right)} \sum_{i=k_{1}}^{\sigma\left(k_{i}\right)-1}(\sigma(i))^{h_{1 \varepsilon}\left(\lambda_{0}\right)+h_{2 \varepsilon}\left(\lambda_{0}\right)} \sum_{j=i}^{+\infty}\left(\sum_{l=1}^{m} p_{l}(j)\left(\tau_{l}(j)\right)^{\lambda_{0}-h_{1 \varepsilon}\left(\lambda_{0}\right)}\right) \leq 1 .
$$

Therefore

$$
\liminf _{k \rightarrow+\infty} k^{-\lambda_{0}-h_{2 \varepsilon}\left(\lambda_{0}\right)} \sum_{i=k_{2}}^{k-1}(\sigma(i))^{h_{1 \varepsilon}\left(\lambda_{0}\right)+h_{2 \varepsilon}\left(\lambda_{0}\right)} \sum_{j=i}^{+\infty}\left(\sum_{l=1}^{m} p_{l}(j)\left(\tau_{l}(j)\right)^{\lambda_{0}-h_{1 \varepsilon}\left(\lambda_{0}\right)}\right) \leq 1 .
$$

Due to the fact that for all sufficiently small $\varepsilon>0$ we have $-\lambda_{0}-h_{2 \varepsilon}\left(\lambda_{0}\right)<0$, the latter inequality obviously implies that

$$
\liminf _{k \rightarrow+\infty} k^{-\lambda_{0}-h_{2 \varepsilon}\left(\lambda_{0}\right)} \sum_{i=1}^{k-1}(\sigma(i))^{h_{1 \varepsilon}\left(\lambda_{0}\right)+h_{2 \varepsilon}\left(\lambda_{0}\right)} \sum_{j=i}^{+\infty}\left(\sum_{l=1}^{m} p_{l}(j)\left(\tau_{l}(j)\right)^{\lambda_{0}-h_{1 \varepsilon}\left(\lambda_{0}\right)}\right) \leq 1
$$


for all sufficiently small $\varepsilon>0$. Taking the upper limit of both sides in the latter inequality as $\varepsilon \rightarrow 0+$, we will obtain the inequality (3.1), where the function $\rho$ is defined by the equalities (3.2), (3.3).

The theorem is proved.

4. Sufficient conditions for oscillation. In this section, using Theorem 3.1, sufficient conditions will be established for oscillation of all proper solutions of the equation (1.1) which generalize the results given in the paper [1].

Theorem 4.1. Let for any $\lambda \in[0,1]$

$$
\limsup _{\varepsilon \rightarrow 0+}\left(\liminf _{k \rightarrow+\infty} \rho(k, \varepsilon, \lambda)\right)>1
$$

where the function $\rho$ is defined by (3.2) with $h_{i \varepsilon}, i=1,2$, defined by (3.3) and $\sigma$ any function satisfying (2.5) (such a funcyion exists due to Lemma 2.5). Then any proper solution of the equation (1.1) is oscillatory.

Proof. Suppose the contrary. Let $u: N_{k_{0}} \rightarrow(0,+\infty)$ with $k_{0} \in N$ be a positive proper solution of the equation (1.1), i.e., $U_{k_{0}} \neq \varnothing$. Taking into account Theorem 3.1, we will conclude that there exists $\lambda_{0} \in[0,1]$ such that the inequality (3.1) holds for $\lambda=\lambda_{0}$. But this contradicts the condition (4.1). The obtained contradiction proves the theorem.

Theorem 4.2. Let $\alpha_{i} \in(0,+\infty), i=1, \ldots, m$, and

$$
\liminf _{k \rightarrow+\infty} \frac{\tau_{i}(k)}{k^{\alpha_{i}}}>0
$$

Then for all proper solutions of (1.1) to be oscillatory it is sufficient that for any $\lambda \in[0,1]$

$$
\limsup _{\varepsilon \rightarrow 0+}\left(\liminf _{k \rightarrow+\infty} k^{-\lambda-h_{2 \varepsilon}(\lambda)} \sum_{i=1}^{k-1} i^{\alpha\left(h_{1 \varepsilon}(\lambda)+h_{2 \varepsilon}(\lambda)\right)} \sum_{j=i}^{+\infty}\left(\sum_{l=1}^{m} p_{l}(j)\left(\tau_{l}(j)\right)^{\lambda-h_{1 \varepsilon}(\lambda)}\right)\right)>1
$$

where

$$
\alpha=\min \left\{1, \alpha_{1}, \ldots, \alpha_{m}\right\}
$$

Proof. To prove the theorem it suffices to show that the conditions of Theorem 4.1 are fulfilled. Due to (4.2) there is $c \in(0,1]$ such that $\tau_{l}(k) \geq c k^{\alpha}, l=1, \ldots, m, k \in N$, where $\alpha$ is given by (4.4). Therefore the function $\sigma(k)=\left[c k^{\alpha}\right]$ satisfies the conditions (2.5). On the other hand, we have

$$
\rho(k, \varepsilon, \lambda)=k^{-\lambda-h_{2 \varepsilon}(\lambda)} \sum_{i=1}^{k-1}\left[c i^{\alpha}\right]^{h_{1 \varepsilon}(\lambda)+h_{2 \varepsilon}(\lambda)} \sum_{j=i}^{+\infty}\left(\sum_{l=1}^{m} p_{l}(j)\left(\tau_{l}(j)\right)^{\lambda-h_{1 \varepsilon}(\lambda)}\right) .
$$


Therefore in view of (3.3) and (4.3) for any $\lambda \in[0,1]$ we have

$$
\begin{aligned}
\sup \lim _{\varepsilon \rightarrow 0+} & \left(\liminf _{k \rightarrow+\infty} \rho(k, \varepsilon, \lambda)\right) \geq \\
& \geq \sup \lim _{\varepsilon \rightarrow 0+}\left(\inf \lim _{k \rightarrow+\infty} k^{-\lambda-h_{2 \varepsilon}(\lambda)} \sum_{i=1}^{k-1} i^{\alpha\left(h_{1 \varepsilon}(\lambda)+h_{2 \varepsilon}(\lambda)\right.} \times\right. \\
& \left.\times \sum_{j=i}^{+\infty}\left(\sum_{l=1}^{m} p_{l}(j)\left(\tau_{l}(j)\right)^{\lambda-h_{1 \varepsilon}(\lambda)}\right)\right) \inf \lim _{\varepsilon \rightarrow 0+} c^{h_{1 \varepsilon}(\lambda)+h_{2 \varepsilon}(\lambda)}>1 .
\end{aligned}
$$

Therefore all the conditions of Theorem 4.1 are fulfilled, which proves our theorem.

Theorem 4.3. Let the conditions (4.2) be fulfilled and for any $\lambda \in[0,1]$

$$
\limsup _{\varepsilon \rightarrow 0+}\left(\liminf _{k \rightarrow+\infty} k^{1-\lambda+\alpha h_{1 \varepsilon}(\lambda)+(\alpha-1) h_{2 \varepsilon}(\lambda)} \times \sum_{j=k}^{+\infty}\left(\sum_{l=1}^{m} p_{l}(j)\left(\tau_{l}(j)\right)^{\lambda-h_{1 \varepsilon}(\lambda)}\right)\right)>\lambda,
$$

where the functions $h_{1 \varepsilon}$ and $h_{2 \varepsilon}$ are defined by (3.3). Then any proper solution of (1.1) is oscillatory.

Proof. To prove the theorem, it suffices to show that (4.5) implies (4.3). In view of (4.5) there exists a sequence $\left\{\varepsilon_{i}\right\}_{i=1}^{+\infty}$ of positive numbers such that $\varepsilon_{i} \rightarrow 0$ as $i \rightarrow+\infty, \delta>0, k_{i} \in N$, $i=1,2, \ldots$, and

$$
\begin{gathered}
k^{1-\lambda+\alpha h_{1 \varepsilon_{i}}(\lambda)+(\alpha-1) h_{2 \varepsilon_{i}}(\lambda)} \sum_{i=k}^{+\infty}\left(\sum_{l=1}^{m} p_{l}(j)\left(\tau_{l}(j)\right)^{\lambda-h_{1 \varepsilon_{i}}(\lambda)}\right) \geq \lambda+\delta, \\
k \in N_{k_{i}}, \quad i=1,2, \ldots
\end{gathered}
$$

Therefore from the equality

$$
\begin{aligned}
& I\left(k, \varepsilon_{i}\right)= k^{-\lambda-h_{2 \varepsilon_{i}}(\lambda)} \sum_{s=1}^{k-1} s^{\alpha\left(h_{1 \varepsilon_{i}}(\lambda)+h_{2 \varepsilon_{i}}(\lambda)\right)} \sum_{j=s}^{+\infty}\left(\sum_{l=1}^{m} p_{l}(j)\left(\tau_{l}(j)\right)^{\lambda-h_{1 \varepsilon_{i}}(\lambda)}\right)= \\
&= k^{-\lambda-h_{2 \varepsilon_{i}}(\lambda)} \sum_{s=1}^{k_{i}-1} s^{\alpha\left(h_{1 \varepsilon_{i}}(\lambda)+h_{2 \varepsilon_{i}}(\lambda)\right)} \sum_{j=s}^{+\infty}\left(\sum_{l=1}^{m} p_{l}(j)\left(\tau_{l}(j)\right)^{\lambda-h_{1 \varepsilon_{i}}(\lambda)}\right)+ \\
&+k^{-\lambda-h_{2 \varepsilon_{i}}(\lambda)} \sum_{s=k_{i}}^{k-1} s^{\alpha\left(h_{1 \varepsilon_{i}}(\lambda)+h_{2 \varepsilon_{i}}(\lambda)\right)} \sum_{j=s}^{+\infty}\left(\sum_{l=1}^{m} p_{l}(j)\left(\tau_{l}(j)\right)^{\lambda-h_{1 \varepsilon_{i}}(\lambda)}\right), \\
& i=1,2, \ldots,
\end{aligned}
$$

we get

$$
I\left(k, \varepsilon_{i}\right) \geq k^{-\lambda-h_{2 \varepsilon_{i}}(\lambda)}(\lambda+\delta) \sum_{s=k_{i}}^{k-1} s^{\lambda-1+h_{2 \varepsilon_{i}}(\lambda)}, \quad i=1,2, \ldots
$$


Thus

$$
\liminf _{k \rightarrow+\infty} I\left(k, \varepsilon_{i}\right) \geq(\lambda+\delta) \inf \lim _{k \rightarrow+\infty} k^{-\lambda-h_{2 \varepsilon_{i}}(\lambda)} \sum_{s=k_{i}}^{k-1} s^{\lambda-1+h_{2 \varepsilon_{i}}(\lambda)}, \quad i=1,2, \ldots
$$

On the other hand, since $\lambda-1+h_{2 \varepsilon_{i}}(\lambda) \leq 0, i=1,2, \ldots$, we have

$$
\begin{aligned}
\sum_{s=k_{i}}^{k-1} s^{\lambda-1+h_{2 \varepsilon_{i}}(\lambda)} & \geq \sum_{s=k_{i}}^{k-1} \int_{s}^{s+1} \xi^{\lambda-1+h_{2 \varepsilon_{i}}(\lambda)} d \xi= \\
& =\int_{k_{i}}^{k} \xi^{\lambda-1+h_{2 \varepsilon_{i}}(\lambda)} d \xi=\frac{1}{\lambda+h_{2 \varepsilon_{i}}(\lambda)}\left(k^{\lambda+h_{2 \varepsilon_{i}}(\lambda)}-k_{i}^{\lambda+h_{2 \varepsilon_{i}}(\lambda)}\right) .
\end{aligned}
$$

Therefore, since $-\lambda-h_{2 \varepsilon_{i}}(\lambda)<0, i=1,2, \ldots$, from (4.6) we get

$$
\inf \lim _{k \rightarrow+\infty} I\left(k, \varepsilon_{i}\right) \geq \frac{\lambda+\delta}{\lambda+h_{2 \varepsilon_{i}}(\lambda)} .
$$

If we pass to upper limit in the latter equality as $i \rightarrow+\infty$, we will obtain

$$
\limsup _{i \rightarrow+\infty}\left(\liminf _{k \rightarrow+\infty} I\left(k, \varepsilon_{i}\right)\right) \geq \frac{\lambda+\delta}{\lambda}>1,
$$

which proves the equality (4.3).

The theorem is proved.

Theorem 4.4. Let the conditions (4.2) hold and for any $\lambda \in[0,1]$

$$
\begin{aligned}
\limsup _{\varepsilon \rightarrow 0+} & \left(\liminf _{k \rightarrow+\infty} k^{1+(\alpha-1)\left(h_{2 \varepsilon}(\lambda)+h_{1 \varepsilon}(\lambda)\right)} \times\right. \\
& \left.\times \sum_{i=k}^{+\infty}\left(\sum_{l=1}^{m} p_{l}(j)\left(\frac{\tau_{l}(i)}{i}\right)^{\lambda-h_{1 \varepsilon}(\lambda)}\right)\right)>\lambda(1-\lambda) .
\end{aligned}
$$

Then any proper solution of (1.1) is oscillatory.

Proof. If $\lambda=0$, then due to the first condition of (3.3), (4.7) clearly implies (4.5). Therefore below we will assume that $\lambda \in(0,1]$ and show that (4.7) implies (4.5). Indeed, from (4.7) it follows that there exist a sequence $\left\{\varepsilon_{i}\right\}_{i=1}^{+\infty}$ of positive numbers satisfying $\varepsilon_{i} \rightarrow 0$ as $i \rightarrow+\infty$, $\delta>0$ and $k_{i} \in N, i=1,2, \ldots$, such that

$$
\begin{gathered}
k^{1+(\alpha-1)\left(h_{2 \varepsilon}(\lambda)+h_{1 \varepsilon_{i}}(\lambda)\right)} \sum_{j=k}^{+\infty}\left(\sum_{l=1}^{m} p_{l}(j)\left(\frac{\tau_{l}(j)}{j}\right)^{\lambda-h_{1 \varepsilon_{i}}(\lambda)}\right)> \\
>\lambda(1-\lambda)(1+\delta), \quad k \in N_{k_{i}}, \quad i=1,2, \ldots
\end{gathered}
$$


On the other hand, by Lemma 2.1, we have

$$
\begin{aligned}
I_{1}\left(k, \varepsilon_{i}\right): & \mathrm{df} \\
= & k^{1-\lambda+\alpha h_{1 \varepsilon_{i}}(\lambda)+(\alpha-1) h_{2 \varepsilon_{i}}(\lambda)} \sum_{j=k}^{+\infty}\left(\sum_{l=1}^{m} p_{l}(j)\left(\tau_{l}(j)\right)^{\lambda-h_{1 \varepsilon_{i}}(\lambda)}\right)= \\
= & k^{1-\lambda+\alpha h_{1 \varepsilon_{i}}(\lambda)+(\alpha-1) h_{2 \varepsilon_{i}}(\lambda)} \sum_{j=k}^{+\infty} j^{\lambda-h_{1 \varepsilon_{i}}(\lambda)}\left(\sum_{l=1}^{m} p_{l}(j)\left(\frac{\tau_{l}(j)}{j}\right)^{\lambda-h_{1 \varepsilon_{i}}(\lambda)}\right)= \\
= & k^{1-\lambda+\alpha h_{1 \varepsilon_{i}}(\lambda)+(\alpha-1) h_{2 \varepsilon_{i}}(\lambda)} k^{\lambda-h_{1 \varepsilon_{i}}(\lambda)} \sum_{j=k}^{+\infty} j^{\lambda-h_{1 \varepsilon_{i}}(\lambda)} \times \\
& \times\left(\sum_{l=1}^{m} p_{l}(j)\left(\frac{\tau_{l}(j)}{j}\right)^{\lambda-h_{1 \varepsilon_{i}}(\lambda)}\right)+ \\
& +k^{1-\lambda+\alpha h_{1 \varepsilon_{i}}(\lambda)+(\alpha-1) h_{2 \varepsilon_{i}}(\lambda)} \sum_{j=k+1}^{+\infty}\left(j^{\lambda-h_{1 \varepsilon_{i}}(\lambda)}-(j-1)^{\lambda-h_{1 \varepsilon_{i}}(\lambda)}\right) \times \\
& \times \sum_{s=j}^{+\infty}\left(\sum_{l=1}^{m} p_{l}(s)\left(\frac{\tau_{l}(s)}{s}\right)^{\lambda-h_{1 \varepsilon_{i}}(\lambda)}\right) \geq \\
\geq & \lambda(1-\lambda)(1+\delta)+\lambda(1-\lambda)(1+\delta) k^{1-\lambda+\alpha h_{1 \varepsilon_{i}}(\lambda)+(\alpha-1) h_{2 \varepsilon_{i}}(\lambda)} \times \\
& \times \sum_{j=k+1}^{+\infty}\left(j^{\lambda-h_{1 \varepsilon_{i}}(\lambda)}-(j-1)^{\lambda-h_{1 \varepsilon_{i}}(\lambda)}\right) j^{-1} j^{(1-\alpha)\left(h_{1 \varepsilon_{i}}(\lambda)+h_{2 \varepsilon_{i}}(\lambda)\right)}
\end{aligned}
$$

$$
\text { for } k \in N_{k_{i}}, \quad i=1,2, \ldots
$$

Since $\alpha \leq 1$, the latter inequality yields

$$
\begin{aligned}
I_{1}\left(k, \varepsilon_{i}\right) \geq & \lambda(1-\lambda)(1+\delta) 1+k^{1-\lambda+h_{1 \varepsilon_{i}}(\lambda)}\left(\frac{k}{k+1}\right)^{(\alpha-1)\left(h_{1 \varepsilon_{i}}(\lambda)+h_{2 \varepsilon_{i}}(\lambda)\right)} \times \\
& \times \sum_{j=k+1}^{+\infty}\left(j^{\lambda-h_{1 \varepsilon_{i}}(\lambda)}-(j-1)^{\lambda-h_{1 \varepsilon_{i}}(\lambda)}\right) j^{-1}
\end{aligned}
$$

$$
\text { for } k \in N_{k_{i}}, \quad i=1,2, \ldots
$$


On the other hand, since the function $k \mapsto \frac{k-1}{k}$ is nondecreasing, we have

$$
\begin{aligned}
\sum_{j=k+1}^{+\infty} & \left(j^{\lambda-h_{1 \varepsilon_{i}}(\lambda)}-(j-1)^{\lambda-h_{1 \varepsilon_{i}}(\lambda)}\right) j^{-1}= \\
& =\sum_{j=k+1}^{+\infty}\left(j-h_{1 \varepsilon_{i}}(\lambda)\right) j^{-1} \int_{j-1}^{j} \xi^{\lambda-h_{1 \varepsilon_{i}}(\lambda)-1} d \xi= \\
& =\left(j-h_{1 \varepsilon_{i}}(\lambda)\right) \sum_{j=k+1}^{+\infty} \frac{j-1}{j(j-1)} \int_{j-1}^{j} \xi^{\lambda-h_{1 \varepsilon_{i}}(\lambda)-1} d \xi \geq \\
& \geq\left(j-h_{1 \varepsilon_{i}}(\lambda)\right) \frac{k}{k+1} \sum_{j=k+1}^{+\infty} \int_{j-1}^{j} \xi^{\lambda-2-h_{1 \varepsilon_{i}}(\lambda)} d \xi= \\
= & \left(j-h_{1 \varepsilon_{i}}(\lambda)\right) \frac{k}{k+1} \int_{k}^{+\infty} \xi^{\lambda-2-h_{1 \varepsilon_{i}}(\lambda)} d \xi= \\
= & \left(j-h_{1 \varepsilon_{i}}(\lambda)\right) \frac{k}{k+1} \frac{k^{\lambda-1-h_{1 \varepsilon_{i}}(\lambda)}}{1-\lambda+h_{1 \varepsilon_{i}}(\lambda)} d \xi .
\end{aligned}
$$

Therefore (4.9) implies

$$
I_{1}\left(k, \varepsilon_{i}\right) \geq \lambda(1-\lambda)(1+\delta)\left(1+\left(\lambda-h_{1 \varepsilon_{i}}(\lambda)\right) \frac{1}{1-\lambda+h_{1 \varepsilon_{i}}(\lambda)}\left(\frac{k}{k+1}\right)^{1+(\alpha-1)\left(h_{1 \varepsilon_{i}}(\lambda)+h_{2 \varepsilon_{i}}(\lambda)\right)} .\right.
$$

Hence it is clear that

$$
\inf \lim _{k \rightarrow+\infty} I_{1}\left(k, \varepsilon_{i}\right) \geq \lambda(1-\lambda)(1+\delta)\left(1+\frac{\lambda-h_{1 \varepsilon_{i}}(\lambda)}{1-\lambda+h_{1 \varepsilon_{i}}(\lambda)}\right), \quad i=1,2, \ldots
$$

Passing to upper limit in this inequality as $i \rightarrow+\infty$, we will get

$$
\limsup _{i \rightarrow+\infty}\left(\liminf _{k \rightarrow+\infty} I_{1}\left(k, \varepsilon_{i}\right)\right) \geq \lambda(1-\lambda)(1+\delta)\left(1+\frac{\lambda}{1-\lambda}\right)=\lambda(1+\delta)>\lambda .
$$

Therefore the inequality (4.5) holds, which proves the validity of the theorem.

Theorem 4.4. immediately implies the following theorem.

Theorem 4.4'. Let the condition (4.2) be fulfilled with $\alpha_{i} \geq 1, i=1, \ldots, m$. Then for any proper solution of (1.1) to be oscillatory it is sufficient that

$$
\limsup _{\varepsilon \rightarrow 0+}\left(\inf \lim _{k \rightarrow+\infty} k \sum_{j=k}^{+\infty}\left(\sum_{l=1}^{m} p_{l}(j)\left(\frac{\tau_{l}(j)}{j}\right)^{\lambda-h_{1 \varepsilon}(\lambda)}\right)\right)>\lambda(1-\lambda) .
$$


Theorem $4.4^{\prime}$ makes Theorem 3.2 of [1] more precise.

Corollary 4.1. Let there exist $\alpha_{l}, l=1, \ldots, m$, such that $\alpha_{l} \in(0,+\infty)$ and

$$
\liminf _{i \rightarrow+\infty} \frac{\tau_{l}(i)}{i}=\alpha_{i}
$$

Then the condition

$$
\inf \lim _{k \rightarrow+\infty} k \sum_{i=k}^{+\infty}\left(\sum_{l=1}^{m} p_{l}(i) \alpha_{l}^{\lambda}\right)>\lambda(1-\lambda)
$$

is sufficient for oscillation of all proper solution of (1.1).

Corollary 4.2. Let the condition (4.11) be fulfilled and there exist $c_{j} \in(0,+\infty), j=1, \ldots, m$ and a function $p: N \rightarrow[0,+\infty)$ such that $p_{j}(k) \geq c_{j} p(k), j=1, \ldots, m$, for large $k$. Then the condition

$$
\liminf _{k \rightarrow+\infty} k \sum_{i=k}^{+\infty} p(i)>\max \left\{\lambda(1-\lambda)\left(\sum_{l=1}^{m} c_{l} \alpha_{l}^{\lambda}\right)^{-1}: \lambda \in[0,1]\right\}
$$

is sufficient for oscillation of all proper solutions of (1.1).

Remark 4.1. Corollaries 4.1 and 4.2 are given in [1]. But the optimality of the condition (4.12) is proved there only in the case $m=1$. Here we will give an example illustrating that the condition (4.12) can not be replaced by the nonstrict inequality for any $m$. Indeed, let $c_{j}$, $\alpha_{j} \in(0,+\infty)$. Denote

$$
c=\max \left\{\lambda(1-\lambda)\left(\sum_{l=1}^{m} c_{l} \alpha_{l}^{\lambda}\right)^{-1}: \lambda \in[0,1]\right\}
$$

and let $\lambda_{0}$ be the point where the right hand side of (4.12) attains its maximum. Consider the equation

$$
\Delta^{2} u(k)+\sum_{j=1}^{m}\left(c \frac{c_{j}}{k^{2}}+\varphi(k)\right) u\left(\left[\alpha_{j} k\right]\right)=0,
$$

where

$$
\varphi(k)=-\frac{-\Delta^{2}\left(k^{\lambda_{0}}\right)+\frac{c}{k^{2}} \sum_{j=1}^{m} c_{j}([\alpha k])^{\lambda_{0}}}{\sum_{j=1}^{m}([\alpha k])^{\lambda_{0}}}
$$

and $[a]$ denotes the integer part of $a$. It is obvious that by (4.15) the function $u=k^{\lambda_{0}}$ is a positive solution of the equation (4.14). Since

$$
\Delta^{2}\left(k^{\lambda_{0}}\right)=\lambda_{0}\left(\lambda_{0}-1\right) k^{\lambda_{0}-2}+k^{\lambda_{0}} o\left(\frac{1}{k^{2}}\right)
$$


by $(4.15)$ we have $\varphi(k)=o\left(\frac{1}{k^{2}}\right)$. Therefore it is obvious that

$$
p_{l}(k)=c \frac{c_{l}}{k^{2}}+\varphi(k) \geq(c-\varepsilon) \frac{c_{l}}{k^{2}}=c_{l} p(k), \quad p(k)=\frac{c-\varepsilon}{k^{2}} .
$$

Since $\sum_{i=k}^{+\infty} i^{-2} \geq k^{-1}$, we have

$$
\inf \lim _{k \rightarrow+\infty} k \sum_{i=k}^{+\infty} p(i) \geq c-\varepsilon
$$

Therefore, due to arbitrariness of $\varepsilon$ we have

$$
\liminf _{k \rightarrow+\infty} k \sum_{i=k}^{+\infty} p(i) \geq c .
$$

On the other hand, since the equation (4.14) has a positive solution, (4.13) and Corollary 4.2 imply

$$
\liminf _{k \rightarrow+\infty} k \sum_{i=k}^{+\infty} p(i) \leq c
$$

Therefore by (4.16) we have $\liminf _{k \rightarrow+\infty} k \sum_{i=k}^{+\infty} p(i)=c$. But this shows that in Corollary 4.2 the inequality (4.12) can not be replaced by the non-strict one.

Corollary 4.3. Let the condition (4.2) be fulfilled, there exist a nonincreasing function $\widetilde{p} \in$ $\in C\left(R_{+} ; R_{+}\right)$and a nondecreasing function $\widetilde{\tau} \in C\left(R_{+} ; R_{+}\right)$such that $\lim _{t \rightarrow+\infty} \tilde{\tau}(t)=+\infty$ and

$$
p_{l}(i) \geq c_{l} \widetilde{p}(i), \quad \tau_{l}(i) \geq d_{l} \widetilde{\tau}(i), \quad l=1, \ldots, m
$$

where $c_{l}, d_{l} \in(0,+\infty)$. Let, moreover, for any $\lambda \in[0,1]$ the condition

$$
\begin{gathered}
\limsup _{\varepsilon \rightarrow 0+}\left(\inf \lim _{k \rightarrow+\infty} k^{1+(\alpha-1)\left(h_{1 \varepsilon}(\lambda)+h_{2 \varepsilon}\right)(\lambda)} \int_{k-1}^{+\infty} \widetilde{p}(1+\xi) \widetilde{\tau}^{\lambda-h_{1 \varepsilon}(\lambda)}(\xi) d \xi\right)> \\
>\lambda(1-\lambda)\left(\sum_{l=1}^{m} c_{l} d_{l}^{\lambda}\right)^{-1}
\end{gathered}
$$

be fulfilled, where $\alpha$ is given by (4.4). Then any proper solution of (1.1) is oscillatory. 
Proof. To prove the corollary, it suffices to show that (4.18) implies (4.7). Indeed, from (4.17) we have

$$
\begin{aligned}
& \sum_{j=k}^{+\infty}\left(\sum_{l=1}^{m} p_{l}(j)\left(\frac{\tau_{l}(j)}{j}\right)^{\lambda-h_{1 \varepsilon}(\lambda)}\right)= \\
& \quad=\sum_{j=k}^{+\infty}\left(\sum_{l=1}^{m} p_{l}(j)\left(\frac{\tau_{l}(j)}{j}\right)^{\lambda-h_{1 \varepsilon}(\lambda)} \int_{j-1}^{j} d s\right) \geq \\
& \geq\left(\sum_{l=1}^{m} c_{l} d_{l}^{\lambda-h_{1 \varepsilon}(\lambda)}\right) \sum_{i=k_{j-1}}^{+\infty} \tilde{p}(1+s) \widetilde{\tau}^{\lambda-h_{1 \varepsilon}(\lambda)}(s) d s= \\
& \quad=\left(\sum_{l=1}^{m} c_{l} d_{l}^{\lambda-h_{1 \varepsilon}(\lambda)}\right) \int_{k-1}^{+\infty} \widetilde{p}(1+s) \widetilde{\tau}^{\lambda-h_{1 \varepsilon}(\lambda)}(s) d s .
\end{aligned}
$$

Therefore (4.18) obviously implies (4.7).

The corollary is proved.

Corollary 4.4. Let $c_{l}, d_{l}, \alpha \in(0,+\infty), l \in 1, \ldots, m$,

$$
p_{l}(i) \geq \frac{c_{l}}{i^{2}}, \quad \tau_{l}(i) \geq d_{l} i^{1+\alpha} .
$$

Then any proper solution of (1.1) is oscillatory.

To prove the corollary, it suffices to note that the conditions of Corollary 4.3 are fulfilled with $\widetilde{p}(t)=\frac{1}{t^{2}}, \widetilde{\tau}(t)=t^{\alpha}$.

Corollary 4.5. Let the conditions (4.2) be fulfilled and there exist nondecreasing functions $\widetilde{\tau}, \widetilde{p} \in C\left(R_{+} ; R_{+}\right)$such that the conditions (4.17) are fulfilled, where $c_{l}, d_{l} \in(0,+\infty), l \in$ $\in 1, \ldots, m$. Let, moreover, for any $\lambda \in[0,1]$ the condition

$$
\begin{gathered}
\sup \lim _{\varepsilon \rightarrow 0+}\left(\inf \lim _{k \rightarrow+\infty} k^{1+(\alpha-1)\left(h_{1 \varepsilon}(\lambda)+h_{2 \varepsilon}(\lambda)\right)} \int_{k}^{+\infty} \widetilde{p}(s) \widetilde{\tau}^{\lambda-h_{1 \varepsilon}(\lambda)}(s) d s\right)> \\
>\lambda(1-\lambda)\left(\sum_{l=1}^{m} c_{l} d_{l}^{\lambda}\right)^{-1}
\end{gathered}
$$

be fulfilled. Then any proper solution of (1.1) is oscillatory.

Corollary 4.6. Let the conditions (4.19) be fulfilled, where $c_{l}, d_{l} \in(0,+\infty), l=1, \ldots, m$, and

$$
p_{l}(i) \geq \frac{c_{l}}{i^{\beta}}, \quad \tau_{l}(i) \geq d_{l} i^{1-\alpha},
$$

where $\beta<2-\alpha, \alpha \in(0,1)$. Then any proper solution of (1.1) is oscillatory. 
1. Koplatadze R., Kvinikadze G., Stavroulakis I. P. Oscillation of second-order linear difference equations with deviating arguments // Adv. Math. Sci. and Appl. Gakötosho, Tokyo. - 2002. - 12, № 1. - P. 217-226.

2. Cheng S. S., Yan T. Ch., Li H. J. Oscillation criteria for second order difference equation // Funkc. ekvacioj. 1991. - 34. - P. $223-239$.

3. Grace A. R., Lalli B.S. Oscillation theorems for second order delay and neutral difference equations // Util. math. - 1994. - 45. - P. $197-211$.

4. Györi I., Ladas G. Oscillation theory of delay differential equations with applications. - Oxford: Oxford Univ. Press, 1991.

5. Hankerson D., Harris G. A. Oscillation of first and second order delay difference equations // Dynam. Syst. and Appl. - 1995. - 4. - P. 251-262.

6. Hinton D. B., Levvis R. T. Spectral analysis of second order difference equations // J. Math. Anal. and Appl. - 1981. - 63. - P. $421-438$.

7. Koplatadze R. Oscillation of linear difference equation with deviating arguments // Comput. Math. Appl. 2001. - 42. - P. $477-486$.

8. Kordonis I.-G. E., Philos Ch. G. Oscillations and nonoscillations in linear delay or advanced difference equations // Math. Comput. Modell. - 1998. - 27, № 7. - P. 11-21.

9. Kwong M.K., Hooker J.M., Patula W.T. Riccati type fronsformation for second order linear difference equations // J. Math. Anal. and Appl. - 1985. - 107. - P. 182-196.

10. Zhang B. G., Tian Ch. J. Oscillation criteria for difference equation with unbounded delay // Comput. Math. Appl. - 1998. - 35, № 4. - P. 19-26.

Received 02.01.09 